\title{
Professor Yumao Du's Experience in Treating Chronic Nephritis
}

\author{
Yang Zhou', Wei Leng2*, Zhifeng Du$^{3}$ \\ ${ }^{1}$ Shaanxi University of Traditional Chinese Medicine, Xianyang 712000, Shaanxi Province, China \\ ${ }^{2}$ The Affiliated Hospital of Shaanxi University of Traditional Chinese Medicine, Xianyang 712000, Shaanxi Province, China \\ ${ }^{3}$ Academic School Inheritance Studio of Shaanxi Du's Kidney Disease, Yumao Hospital, Xianyang 712000, Shaanxi \\ Province, China \\ *Corresponding author: Wei Leng, lwselt@126.com

\begin{abstract}
Chronic nephritis is a common clinical kidney disease, with insidious onset and long course, which can eventually develop into chronic renal failure. In Chinese medicine, it belongs to the categories of "edema," "back pain," "deficiency," and "vertigo." Professor Du Yumao is a well-known Chinese medicine doctor and a well-known nephropathy expert in China. He has rich clinical experience and unique insights into the treatment of chronic nephritis. He believes that insufficient kidney yin is the key to this disease, and water dampness and blood stasis are important pathological factors. Appropriate change of method according to the situation and flexible use of medicine often lead to good clinical outcomes.
\end{abstract}

Keywords: Yumao Du; Chronic nephritis; Clinical experience

Publication date: July 2021; Online publication: July 30, 2021

\section{Introduction}

Chronic nephritis is a common clinical disease in nephrology. It is a glomerular disease composed of different pathological types. Its main clinical manifestations are proteinuria, hematuria, edema, and hypertension, etc. Chronic nephritis is mostly accompanied by insidious onset, but the condition is persistent and difficult to heal. Eventually, it may develop into chronic renal failure ${ }^{[1]}$. At present, for the treatment of chronic glomerulonephritis, Western medicine generally uses drugs such as angiotensinconverting enzyme inhibitor (ACEI) or angiotensin receptor blocker (ARB), hormones and immunosuppressive agents to achieve certain effects and help relieve symptoms, but there are also certain side effects. In addition to conventional Western medicine treatment, Chinese medicine treatment is also the focus of clinical attention. Professor Yumao Du has studied Qihuang and learned profound skills. He has deep attainments in Chinese medicine theory and treatment, as well as unique insights into the treatment of chronic nephritis. This article presents our summarizations based on Professor Du's old medical records and collections of experience.

\section{Awareness of etiology and pathogenesis}

Professor Du believes that the etiology and pathogenesis of the disease are quite complicated, either due to wind-heat external attack, or dampness and turbidity, or excessive fatigue, or visceral disorders, but its onset is hidden, and there are often mistakes in treatment. The history of treatment has caused a lingering course of disease and aggravated and prolonged disease. Regarding the location, Professor Du believes that the kidneys are first to suffer from this disease. According to The Origin of Chao's Disease, "the kidneys dominate water, and kidney deficiency cannot produce water, so the water overflows into the skin and the 
body becomes swollen." It also involves the lungs, spleen, liver, and triple burnt organs. Due to the dysfunction of the viscera, the $q i$ resolves the loss of division, puts a stop to the accumulation of water and dampness, turns heat from stagnation, consumes yin fluid, and internal resistance of blood stasis. The four evils, i.e. dampness, heat, blood stasis, and toxin, combine with each other as pathological factors that affect the function of viscera and contribute to pathogenesis of chronic nephritis based on a mixture of deficiency and excess. However, Professor Du believes that the core is still based on deficiency, especially the lack of kidney yin as the root cause, and the focus is on water dampness and blood stasis as the standard ${ }^{[2]}$.

\section{Experience in medication and treatment based on syndrome differentiation \\ 3.1. The triple focus is degraded and the gasification is not good}

The onset of chronic nephritis is often due to the failure of the Sanjiao Judu, and the gasification of the bladder is unfavorable, which leads to dampness and internal stagnation. The clinical manifestations showed high edema of the whole body, which has the touch of mud when pressed, chest tightness, swelling, poor urination, fat tongue with tooth marks, and greasy moss. Professor Du believes that the key to the pathogenesis of this syndrome lies in the inability of yang $q i$ and the cessation of water dampness ${ }^{[3]}$. The treatment should be the method of promoting yang and dampness. As a result, the $q i$ machine is unblocked, the yang $q i$ is unblocked, and the triple-burner and bladder would gasify as usual. The waterway is open, and the water is wet. Commonly used drugs include big belly peel, tangerine peel, cassia twig, ginger peel, etc. Secondly, the water can penetrate from the lower Jiao, such as Polyporus umbellatus, Poria cocos, Alisma, etc.

\subsection{Loss of kidney yin, and stagnation of water and dampness}

In addition to the manifestations of swelling and depression of the whole body, there are five signs of upset heat, soreness of the waist and knees, nocturnal emission, tinnitus, night hot flashes and night sweats, red tongue, and few pulses. The true yin in the kidney is the source of yin and fluid in a person's body. The patient's factors include body yin deficiency, or chronic illness and damage to yin. Based on this, Professor Du believes that mere water diuresis may hurt the true yin, nourish yin and clear away heat, and accumulate dampness; therefore, it is advisable to use the method of nourishing yin and removing dampness, in accordance with Zhulingtang in Treatise on Febrile Diseases ${ }^{[4]}$. Good results could be obtained using Liuwei Dihuang Wan with or without Polyporus umbellatus Decoction.

\subsection{Insufficient kidney yin and loss of yang qi}

However, chronic nephritis may persists and is difficult to heal. It can cause yin and yang for a long time. It often causes both yin and yang. In addition to the manifestations of kidney insufficiency and yin, there are also severe swelling below the waist, cold limbs, frequent nocturia, pale red tongue and white fur. The pulse is thin and the string is weak. Kidney is the viscera of water and fire, where yin and yang mutually use and complement each other. To nourish yin and take six flavors, warming yang is better than aconite cinnamon. Jinkui Shenqi pills are commonly used in clinical practice to supplement yin and yang. Yin and yang have an inexhaustible source, and will metamorphose infinitely ${ }^{[4]}$.

\subsection{Deficiency of spleen and dampness}

The spleen is in the middle of the state, preferring dryness but repelling dampness. If the spleen body is weak, or the spleen is injured by long-term illness, the spleen is in insufficiency, the movement is lost, and the dampness stops. The syndrome is common with body edema, depression, dizziness, shortness of breath, fatigue, pale lips, pale tongue, white and moist coating, and weak pulse. Professor Du believes that although the edema is obvious, the root of the syndrome lies in the failure of the spleen to strengthen the spleen ${ }^{[5]}$. 
Therefore, the key to the treatment is not to promote water but to strengthen the spleen. Therefore, the method of replenishing $q i$ and strengthening the spleen should be used. Atractylodes, Codonopsis, Chinese yam, etc. nourish the temper, and the other is to invigorate the spleen and help transport. The spleen is the pivot of the qi machine. Tangerine peel, atractylodes, Magnolia officinalis, hawthorn, etc. are used to transport the spleen, so that the edema disappears.

\subsection{Intrinsic dampness, blood stasis and collaterals}

Chronic illnesses and blood stasis are common aspects in Chinese medicine. In the middle and late stages of chronic nephritis, it often progresses to internal resistance of blood stasis. The internal stagnation due to water and dampness blocks the menstrual flow, blood stasis is caused by poor blood flow, and the mutual dampness and blood stasis aggravate the condition. This syndrome is characterized by persistent edema, low back pain such as thorns, dark lips, dark purple tongue, petechiae, and astringent pulse. In response to this situation, the method of promoting blood circulation and diuresis is needed to promote blood circulation to remove blood stasis and to relieve swelling. Professor Du often adds motherwort ${ }^{[6]}$ to the medicine for differentiation of symptoms and signs, the dosage is about $30 \mathrm{~g}$, and heavy doses are used to cause swelling. Motherwort is bitter in nature, slightly cold, and returns to the liver. The kidney meridian is especially effective in promoting blood circulation, regulating menstruation, hydration and reducing swelling, and the following prescriptions have been used in combination with reliable results. In this disease, blood stasis and water coexist.

\section{Conclusion}

Chronic nephritis has a long course, and it is difficult to heal after prolongation. In the decades of clinical experience, Professor Du has gained some unique experience in the treatment of chronic nephritis. He believes that the disease is based on deficiency, damp-heat and blood stasis as the standard, and it is based on water dampness and blood stasis as the most important treatment. Methods such as yang and dampness, nourishing yin and diuresis, tonifying yin and yang, replenishing $q i$ and invigorating the spleen promote blood circulation and diuresis. Flexible prescriptions according to the syndromes may lead to good results. In the treatment of chronic nephritis, we believe flexible use of traditional Chinese medicine on the basis of Western medicine treatments can be further improved by their combination. We look forward to further progress in the treatment of chronic nephritis, which can effectively delay the progression of the disease and reducing the burden on patients.

\section{Funding}

Shaanxi Du's Nephropathy Academic School Inheritance Studio Project (Shaanxi Traditional Chinese Medicine [2018] No. 40); Research Project of Shaanxi Administration of Traditional Chinese Medicine (2019-ZZ-LC050)

\section{Disclosure statement}

The authors declare that there is no conflict of interest.

\section{References}

[1] Wang XQ, Shao CD, Ba YM, 2011, Guidelines for the Diagnosis and Treatment of Chronic Glomerulonephritis. Modern Distance Education of Chinese Medicine, 9(09): 129-132.

[2] Zhang XK, Zhang J, 1993, Professor Du Yumao's Experience in Treating Chronic Nephritis with 
Jingfang. Shaanxi Journal of Traditional Chinese Medicine, (11): 19-20+22.

[3] Guo JL, Yang YG, 1991, Summary of Professor Du Yumao's Experience in Treating Chronic Nephritis. Heilongjiang Traditional Chinese Medicine, 1991(04): 1-4+56.

[4] Zhang XK, Du YM, 1990, Experience in Treating Chronic Nephritis from Nourishing Yin Jing. Hubei Journal of Traditional Chinese Medicine, 1990(02): 2-3.

[5] Zhang XKi. 1989, Professor Du Yumao's Eight Methods of Treating Chronic Nephritis. Jiangsu Traditional Chinese Medicine, 1989(12):1-3.

[6] Zhang YQ, Guo JL, 1991, Extracts of Professor Du Yumao's Medications for Treating Nephritis. Forum on Traditional Chinese Medicine, 1991(03): 19-21. 